\title{
RENDIMIENTO ACADÉMICO, ESTRÉS Y ESTRATEGIAS DE AFRONTAMIENTO EN ALUMNOS DEL PROGRAMA DE ALTA EXIGENCIA ACADÉMICA DE LA CARRERA DE MEDICINA
}

\author{
Silvia Ortiz ${ }^{*}$, Silvia Tafoya², Aurora Farfán ${ }^{3}$, Aurora Jaimes \\ ${ }^{1}$ Médico Psiquiatra y Psiquiatra de niños y adolescentes. Departamento de Psiquiatría y Salud Mental. \\ Universidad Nacional Autónoma de México \\ ${ }^{2}$ Psicóloga.MSc Psicología General Experimental. Departamento de Psiquiatría y Salud Mental. \\ Universidad Nacional Autónoma de México. \\ ${ }^{3}$ Etnología. MSc Salud Pública. Universidad Nacional Autónoma de México. \\ ${ }^{4}$ Psicóloga. MSc Psicobiología. Departamento de Psiquiatría y Salud Mental. Universidad Nacional Autónoma de México. \\ *Correspondencia:dra_silviaortiz@yahoo.com.mx
}

Recibido: Agosto 1 de 2012 Aceptado: Octubre 22 de 2012

\begin{abstract}
Resumen
Vincular las fuentes de estrés, el afrontamiento y el desempeño académico en los estudiantes de medicina. Para ésto se llevó a cabo un estudio longitudinal en el que participaron 93 estudiantes en el primer año y 80 estudiantes de segundo año en el seguimiento, pertenecientes a un programa de alto desempeño académico de la carrera de medicina. Se aplicó el Cuestionario de Fuentes de Estrés en Estudiantes de Medicina y el Cuestionario de Estrategias de Afrontamiento en cuatro momentos (al inicio y al final del primero y segundo años). Se observó que el nivel de estrés se mantuvo sin cambios significativos para primero y segundo años, tanto en la primera como segunda evaluación $(F=2.18$, $\mathrm{p}=0.09$ ). Sin embargo, sólo el puntaje de estrés detectado en la primera medición del primer año se relacionó de forma significativa con el promedio final de este año escolar $(\mathrm{r}=-0.33, \mathrm{p} \leq 0.05)$. El afrontamiento predominante en ambos años de la carrera de medicina fue la estrategia de "Solución de problemas" y la menos utilizada fue el "Distanciamiento". Al comparar las estrategias de afrontamiento entre los estudiantes de primero y segundo años, se observaron diferencias en el uso del "distanciamiento", que fue más utilizado por los alumnos en segundo año y el "apoyo social" por los alumnos en primero. A pesar de que las calificaciones fueron menores en los estudiantes cuya estrategia de afrontamiento predominante fue el "pensamiento mágico" y los niveles de estrés más altos, no hubo diferencias significativas con respecto a las otras estrategias. Se concluyó, que los niveles de estrés permanecen constantes en los alumnos de primero y segundo años de la carrera de medicina, pero las fuentes que lo generan y el modo de afrontarlas, se modifican conforme transcurre el ciclo escolar y parecen asociarse al desempeño académico de esta población.
\end{abstract}

Palabras clave: Adaptación psicológica, fuentes de estrés, alumnos de medicina, programa de alto desempeño académico.

\section{ACADEMIC PERFORMANCE, STRESS AND COPING STRATEGIES AMONG MEDICAL STUDENTS ON THE HIGH ACADEMIC PERFORMANCE PROGRAM.}

\begin{abstract}
To link the sources of stress, coping strategies and academic performance among medical students. For this we conducted a longitudinal study involving 93 first-year undergraduates and with 80 second-year
\end{abstract}


undergraduates in the follow up, enrolled on the Faculty of Medicine's high academic performance program. Two surveys-Sources of Stress among Medical Students and Coping Strategies-were carried out at four stages (at the start and end of the first and second years). It was observed that the stress level was maintained without significant changes to the first and second year, both at the first and at the second evaluation $(\mathrm{F}=2.18, \mathrm{p}=0.09)$. However, only the stress scores detected at the initial measurement of the first year was related significantly to the final average of that year $(r=-0.33$, $\mathrm{p} \leq 0.05$ ). In both year groups of medical students, "Problem Solving" was revealed as the main coping strategy, while "Distancing" was the strategy least used. By comparing the coping strategies between first and second-year students, differences were detected in the use of "Distancing," which was more prevalent among second-year students while "Social Support" was used more among first-year students. Although students whose coping strategy was mainly "Wishful Thinking" scored lower and experienced higher stress levels, no significant differences were found compared to other strategies. Was concluded that stress levels remained constant among first and second-year medical students but the sources that create them and the coping strategies change over the school year and appear linked to this population's academic performance.

Keys words: Coping behavior, sources of stress, medical students, high academic performance program.

\title{
RENDIMENTO ACADÊMICO, ESTRESSE E ESTRATÉGIAS DE AFRONTAMENTO EM ALUNOS DO PROGRAMA DE ALTA EXIGÊNCIA ACADÊMICA DO CURSO DE MEDICINA
}

\begin{abstract}
Resumo
Vincular as fontes de estresse, o afrontamento e o desempenho acadêmico nos estudantes de medicina. Para isto levou-se a cabo um estudo longitudinal no qual participaram 93 estudantes no primeiro ano e 80 estudantes de segundo ano no seguimento, pertencentes a um programa de alto desempenho acadêmico do curso de medicina. Aplicou-se o Questionário de Fontes de Estresse em Estudantes de Medicina e o Questionário de Estratégias de Afrontamento em quatro momentos (ao início e ao final do primeiro e segundo anos). Observou-se que o nível de estresse se manteve sem câmbios significativos para o primeiro e segundo anos, tanto na primeira quanto segunda avaliação $(\mathrm{F}=2.18, \mathrm{p}=0.09)$. Porém, só a contagem de estresse detectada na primeira medição do primeiro ano se relacionou de forma significativa com a média final deste ano escolar $(\mathrm{r}=-0.33, \mathrm{p} \leq 0.05)$. O afrontamento predominante nos dois anos do curso de medicina foi a estratégia de "Solução de problemas" e a menos utilizada foi o "Distanciamento". Ao comparar as estratégias de afrontamento entre os estudantes de primeiro e segundo anos, se observaram diferenças no uso do "distanciamento", que foi mais utilizado pelos alunos em segundo ano e o "apoio social" pelos alunos em primeiro. A pesar de que as qualificações foram menores nos estudantes cuja estratégia de afrontamento predominante foi o "pensamento mágico" e os níveis de estresse mais altos, não houve diferenças significativas com respeito às outras estratégias. Concluiu-se, que os níveis de estresse permanecem constantes nos alunos de primeiro e segundo anos do curso de medicina, mas as fontes que o geram e o modo de afrontá-las, modificam-se conforme transcorre o ciclo escolar e parecem associar-se ao desempenho acadêmico desta população.
\end{abstract}

Palavras chave: Adaptação psicológica, fontes de estresse, alunos de medicina, programa de alto desempenho acadêmico. 


\section{Antecedentes}

La literatura ha descrito a la carrera de medicina como altamente demandante y por tanto generadora de estrés entre sus estudiantes. Pese a que éste se presenta de manera cotidiana durante su formación, para algunos estudiantes representa un reto, mientras que para otros se constituye como una amenaza, ya que provoca sentimientos de incompetencia, frustración y enojo, que repercuten negativamente en su salud física y psicológica (1-3). Dentro del plan curricular se creó un programa con el fin de alcanzar un alto nivel de calidad educativa, tal es el caso del Programa de Alta Exigencia Académica (PAEA), en el que los alumnos deben de cumplir con el plan curricular de la carrera y simultáneamente llevar a cabo otras habilidades que incluyen la iniciación temprana a la investigación, aprendizaje basado en problemas, cursos clínicos y sesiones de asesoría personalizada. Todas estas actividades representan una carga adicional de trabajo académico, a la que se suma la exigencia de mantener un promedio mínimo de ocho en cada asignatura para permanecer dentro del programa y a su vez genera condiciones mayormente estresantes en los estudiantes.

Entre los principales factores estresantes, asociados a la formación médica, se encuentran la sobrecarga académica así como el contacto con la enfermedad y la muerte (4); algunos autores enfatizan los aspectos académicos como la principal fuente de estrés, ya que de ellos se deriva la sobrecarga de trabajo, temor a obtener bajas calificaciones y no contar con el tiempo suficiente tanto para estudiar todos los contenidos de las asignaturas, como para las actividades recreativas (5). Otros autores reportan otras fuentes adicionales de estrés, que pueden influir sobre el desempeño académico, como: la situación económica, la familia y la percepción de su desempeño (6-9). No obstante, algunos estudiantes cuentan con los suficientes recursos personales para afrontar las dificultades, pero otros requieren de apoyos como los programas que fomentan estrategias de afrontamiento, cuyo objetivo principal es el entrenamiento en el manejo adecuado de las condiciones de estrés $(10,11)$.

Un estudio sobre los mecanismos de afrontamiento ante el estrés en los estudiantes de medicina, encontró que éstos son diferentes dependiendo del año escolar y del tipo de estresor que experimenta el estudiante. Las estrategias de afrontamiento que utilizan ante el estrés puede tener un resultado positivo o negativo en ellos, por ejemplo cuando utilizan estrategias de afrontamiento centradas en la evitación como el distanciamiento social, el pensamiento mágico y la autocrítica, tienen mayores consecuencias negativas como depresión, ansiedad y percepción de una salud mental deficiente. En contraste, los que utilizan estrategias de solución de problemas, reinterpretación positiva, apoyo social y expresión de la emoción, responden de una manera adaptativa y por consiguiente reducen los efectos del estrés en su salud física y mental (12).

En cuanto a los estilos de afrontamiento utilizados de acuerdo al sexo, los estudios reportan que las mujeres tienden a utilizar con mayor frecuencia estilos dirigidos a la emoción, como la búsqueda de apoyo social o la evitación (13); mientras que los hombres presentan estilos más enfocados a la solución del problema (14). Es importante señalar que en los universitarios, los estilos de afrontamiento también se relacionan con las fuentes de estrés que son diferentes para hombres y mujeres (15). Por otro lado, algunos autores señalan que en los hombres universitarios pueden cambiar su estilo de afrontamiento dirigido a la solución de problemas por el estilo que se enfoca en la emoción, como consecuencia de los cambios de residencia, la convivencia con sus pares y la vida cotidiana (16).

Todo lo anterior cobra importancia, debido al impacto que puede tener la modificación de las fuentes de estrés y los estilos de afrontamiento, sobre el desempeño académico en los dos primeros años de la carrera de medicina. Por lo que se evaluaron las fuentes de estrés, las estrategias de afrontamiento y el rendimiento académico en los estudiantes de medicina del PAEA, en los dos primeros años con el fin de analizar su asociación.

\section{Método}

\section{Participantes}

En el primer año participaron 93 estudiantes del Programa de Alta Exigencia Académica (PAEA) de la carrera de medicina, y en el seguimiento del segundo año participaron 80 estudiantes.

\section{Procedimiento}

Estudio longitudinal, aceptado por las comisiones de investigación y ética locales. La participación fue voluntaria y quienes aceptaron, firmaron una carta de consentimiento informado. Los instrumentos aplicados 
RENDIMIENTO ACADÉMICO, ESTRÉS Y ESTRATEGIAS DE AFRONTAMIENTO EN ALUMNOS

del Programa de Alta Exigencia Académica de la Carrera de Medicina

fueron la Escala de Fuentes de Estrés de Estudiantes de Medicina y el Cuestionario de Estrategias de Afrontamiento (Maupone y Sotelo), cuyo tiempo de aplicación fue de 30 minutos aproximadamente. Las evaluaciones se realizaron en cuatro momentos: al inicio y al final del primero y segundo años (la primera un mes después del inicio del año escolar y la segunda un mes antes de su conclusión). Las calificaciones del rendimiento académico se obtuvieron una vez que se terminó el primero y segundo año escolar.

\section{Instrumentos}

Cuestionario de Fuentes de Estrés en Estudiantes de Medicina: este instrumento consta de 25 preguntas referentes a situaciones de estrés propias de la carrera de medicina, cuya última opción es abierta para que el alumno comente alguna circunstancia no contemplada dentro del instrumento. Se responde con base a una escala tipo Likert de acuerdo a la severidad de la tensión que cada situación le ocasiona, y va de " $1=$ No le ocurrió/No le tensó" a " $5=$ Tensión severa". $\mathrm{El}$ cuestionario se puede analizar por reactivo y por puntaje total. La consistencia interna del instrumento reportada por Fouilloux y colaboradores 5 , fue de un $\alpha$ de Cronbach de 0.85. Y para este estudio se encontró una consistencia interna similar ( $\alpha$ de Cronbach $=0.87$ ).

Cuestionario de Modos de Afrontamiento (Sotelo y Maupone): se utilizó una versión en español validada y estandarizada en jóvenes de 15 a 24 años con 66 reactivos clasificados en seis factores: 1 . Flexibilidad de afrontamiento $(\alpha=0.90), 2$. Afrontamiento dirigido al problema $(\alpha=0.63), 3$. Pensamiento Mágico $(\alpha=0.73), 4$. Distanciamiento ( $\alpha=0.59), 5$. Búsqueda de apoyo social $(\alpha=0.84)$ y 6 . Revaloración Positiva ( $\alpha$ de Cronbach $=0.57$ ); la confiabilidad reportada para la escala total tuvo un $\alpha$ de Cronbach $=0.9017$. La calificación es por subescalas, en donde a cada una se le otorga un porcentaje ponderado respecto al uso del modo de afrontamiento. Para este estudio, se obtuvieron dos calificaciones de afrontamiento: una cuantitativa, correspondiente al porcentaje de utilización de cada modo de afrontamiento; y otra cualitativa, en la que se describió el tipo de afrontamiento "predominante" de cada estudiante. Al igual que lo observado por Sotelo y Maupone, en el presente estudio, la escala total presentó un $\alpha$ de Cronbach $=0.90$.

\section{Análisis Estadístico}

Los datos se analizaron con el paquete estadístico PASW-18 (SPSS Inc. Chicago, IL). La captura de la información se validó a través de análisis descriptivos. La descripción de los datos sociodemográficos se hizo con frecuencias y promedios de acuerdo al nivel de medición de las de variables. Para la medición de las fuentes de estrés se obtuvo el puntaje promedio y la desviación estándar por reactivo y por la escala total. Para el afrontamiento se obtuvieron dos calificaciones: una correspondiente al porcentaje de utilización de cada tipo de afrontamiento (porcentaje promedio) y otra del afrontamiento predominante (frecuencias). La comparación de los puntajes de estrés por reactivo y por escala total en los diferentes momentos se realizó mediante la prueba de Kruskal-Wallis (por reactivo) y con un ANOVA (por escala total). El puntaje total de estrés y los promedios de cada reactivo se correlacionaron con la calificación final del primero y segundo años mediante el rho de Spearman. Por último se obtuvieron los promedios y desviaciones estándar de la calificación final de cada año académico y del puntaje total de estrés, de acuerdo con la estrategia de afrontamiento predominante, con el fin de comparar los grupos.

\section{Resultados}

De los 106 estudiantes que conformaron la población del PAEA, sólo aceptaron participar 93 en el primer año, y cuyo promedio de edad para ese momento fue de 18.62 años $(\mathrm{DE}=0.99), 50(54 \%)$ hombres y $43(46 \%)$ mujeres, todos ellos solteros. En el segundo año, de los 93 participantes iniciales, sólo 86 alumnos continuaron dentro del programa y 80 aceptaron participar, 47 (59\%) hombres y 33 (41\%) mujeres, que mantuvieron su estado civil. No se observaron diferencias entre hombres y mujeres, en los puntajes de estrés ni en las subescalas de afrontamiento tanto del primer y segundo año.

\section{Estrés y rendimiento académico}

El nivel de estrés se mantuvo sin cambios significativos para primero y segundo años, tanto en la primera como segunda evaluación $(F=2.18, p=0.09)$. Sin embargo, sólo el puntaje estrés detectado en la primera medición del primer año se relacionó de forma significativa con el 
promedio final de este año escolar $(\mathrm{r}=-0.33, \mathrm{p} \leq 0.05)$. A diferencia de los resultados del segundo año, en los que no se observó relación entre los puntajes de estrés con las calificaciones finales correspondientes a este año.

En cuanto al malestar relacionado con las fuentes de estrés, se observó que conforme los estudiantes avanzaron en los ciclos académicos, el malestar aumentaba ante las siguientes fuentes de estrés: "proximidad de los exámenes departamentales", "temor a obtener malas calificaciones" y "no poder definir cuáles eran los conocimientos más relevantes". Por otro lado, los puntajes de la fuente de estrés relacionada con "dudas vocacionales", fueron más altos al inicio del primer año y más bajos al final del mismo. El cuadro 1 muestra el malestar promedio para cada fuente de estrés en los alumnos del PAEA, en primero y segundo años.

La relación entre el malestar de cada fuente de estrés y la calificación final correspondiente a cada año escolar,

Cuadro 1. Cambios en el malestar promedio para cada una de las fuentes de estrés en los alumnos del paea en primero (2008) y segundo (2009) años.

\begin{tabular}{|c|c|c|c|c|c|}
\hline & \multicolumn{2}{|c|}{ Primero } & \multicolumn{2}{|c|}{ Segundo } & \multirow{2}{*}{$\mathbf{p}$} \\
\hline & Inicio & Final & Inicio & Final & \\
\hline Proximidad de los exámenes departamentales & $1.80(1.13)^{*}$ & $3.24(0.95)$ & $3.49(0.73)$ & $3.30(1.01)$ & 0.0001 \\
\hline Sensación de incapacidad para absorber todo el material del curso & $2.48(0.93)^{*}$ & $2.88(0.95)$ & $3.33(1.01)$ & $2.92(1.13)$ & 0.002 \\
\hline No poder definir cuáles son los conocimientos más relevantes & $1.84(0.82)^{*}$ & $2.22(1.05)$ & $2.71(1.06)$ & $2.20(1.09)$ & 0.002 \\
\hline Temor a obtener malas calificaciones & $2.42(0.89) *$ & $2.81(1.20)$ & $3.06(1.14)$ & $3.15(1.00)$ & 0.01 \\
\hline Desconocimiento del método de estudio & $1.84(0.82)$ & $2.14(1.09)$ & $2.41(1.10)$ & $2.00(1.03)$ & 0.08 \\
\hline No mantenerse firme en el tiempo que debería dedicarle al estudio & $2.29(1.07)$ & $2.54(1.10)$ & $2.80(1.07)$ & $2.82(1.22)$ & 0.10 \\
\hline Necesidad de manejo de otro idioma & $1.87(0.96)$ & $1.58(0.88)$ & $1.94(0.90)$ & $1.85(0.98)$ & 0.09 \\
\hline $\begin{array}{l}\text { Tener que limitar las actividades recreacionales y los compromisos } \\
\text { sociales }\end{array}$ & $2.90(1.01)$ & $2.47(1.15)$ & $2.94(1.17)$ & $2.54(1.13)$ & 0.09 \\
\hline Dudas vocacionales & $2.23(1.23)$ & $1.58(1.07)^{*}$ & $2.45(1.39)$ & $1.70(1.01)$ & 0.0001 \\
\hline Exponer ante compañeros y maestros & $1.87(0.67)$ & $1.73(0.91)$ & $2.06(0.93)$ & $1.92(0.94)$ & 0.17 \\
\hline Falta de tiempo para encuentros sexuales & $1.90(1.27)$ & $1.34(0.73)^{*}$ & $1.86(1.20)$ & $1.90(1.24)$ & 0.02 \\
\hline Sensación de aislamiento dentro de la Facultad & $1.71(0.86)$ & $1.71(0.93)$ & $2.00(1.10)$ & $1.81(1.04)$ & 0.57 \\
\hline Trámites administrativos de la Facultad & $2.19(1.01)$ & $1.68(1.01)^{*}$ & $2.14(1.28)$ & $1.80(1.03)$ & 0.04 \\
\hline Temor durante las primeras experiencias de disección con un cadáver & $1.30(0.54)$ & $1.53(0.84)$ & $1.39(0.67)$ & $1.48(0.73)$ & 0.61 \\
\hline Problemas de relación interpersonal con los compañeros & $1.77(0.56)$ & $1.42(0.70)^{*}$ & $1.64(0.94)$ & $1.65(0.88)$ & 0.04 \\
\hline Problemas de relación interpersonal con los profesores & $1.71(0.53)$ & $1.53(0.68)$ & $1.65(0.72)$ & $1.59(0.79)$ & 0.40 \\
\hline $\begin{array}{l}\text { Experimentar conflicto ante la dependencia familiar y la necesidad } \\
\text { personal de independencia }\end{array}$ & $1.94(1.12)$ & $1.69(1.06)$ & $2.00(1.17)$ & $1.67(0.98)$ & 0.20 \\
\hline Ser consultado como médico durante el primer año de la carrera & $1.52(0.81)$ & $1.93(0.83)$ & $1.71(0.94)$ & $1.82(0.93)$ & 0.09 \\
\hline Temor al fallecimiento de un paciente & $1.90(1.27)$ & $1.56(0.82)$ & $1.63(0.96)$ & $1.67(0.98)$ & 0.85 \\
\hline Temor a equivocarse en el diagnóstico o tratamiento & $1.93(1.23)$ & $1.46(0.77)$ & $1.88(1.19)$ & $1.94(1.16)$ & 0.09 \\
\hline Temor a no saber manejar pacientes con enfermedades incurables & $1.74(1.15)$ & $1.34(0.78)^{*}$ & $1.82(1.14)$ & $1.73(1.07)$ & 0.04 \\
\hline Temor a perder la salud por estar en contacto con enfermos & $1.42(0.72)$ & $1.37(0.74)$ & $1.65(1.00)$ & $1.63(0.91)$ & 0.30 \\
\hline $\begin{array}{l}\text { Temor a hablar con pacientes acerca de sus problemas conyugales } \\
\text { o sexuales }\end{array}$ & $1.16(0.37)$ & $1.19(0.51)$ & $1.28(0.61)$ & $1.35(0.68)$ & 0.27 \\
\hline Temor a explorar a pacientes del sexo opuesto & $1.30(0.61)$ & $1.11(0.37)$ & $1.23(0.53)$ & $1.33(0.66)$ & 0.14 \\
\hline Temor a explorar a pacientes del mismo sexo & $1.39(0.72)$ & $1.09(0.35)^{*}$ & $1.39(0.72)$ & $1.28(0.56)$ & 0.03 \\
\hline
\end{tabular}

Nota: En la medida en que los puntajes promedios se elevan indican una mayor percepción de estrés.

* Las marcas evidencian los valores más bajos en las evaluaciones. 
mostró que: el "temor a obtener malas calificaciones" en la primera y segunda evaluación de primer año presentó una correlación negativa con la calificación final de este año $(r=-0.37, p \leq 0.01$ y $r=-0.37, p \leq 0.05)$. También se observó, que tanto en la primera como en la segunda evaluación del primer año, a medida que aumentaba el malestar respecto a "tener que limitar las actividades recreacionales y los compromisos sociales" el promedio de las calificaciones finales disminuía ( $\mathrm{r}=$ $0.42, \mathrm{p} \leq 0.01$ y $\mathrm{r}=-0.26, \mathrm{p} \leq 0.05)$. El malestar por la "proximidad de exámenes departamentales" al final del primer año, se relacionó de forma negativa con el promedio de este año $(\mathrm{r}=-0.30, \mathrm{p} \leq 0.05)$. La "sensación de incapacidad para absorber todo el material del curso", en la segunda evaluación del primero y segundo años, tuvo una relación negativa con el promedio correspondiente de ambos $(\mathrm{r}=-0.27, \mathrm{p} \leq 0.05)$. Del mismo modo, al final del segundo año el "temor a perder la salud por estar en contacto con enfermos", se relacionó de forma negativa con el promedio de calificaciones de este año escolar $(r=-0.24, p \leq 0.05)$.

\section{Estrés, tipo de afrontamiento y rendimiento académico}

El afrontamiento predominante en ambos años de la carrera de medicina fue la estrategia de "Solución de problemas" y la menos utilizada fue el "Distanciamiento". Sin embargo, cuando se compararon las estrategias de afrontamiento entre los estudiantes de primero y segundo año, se observaron diferencias en el uso del "distanciamiento", que fue más utilizado por los alumnos en segundo año y el "apoyo social" por los alumnos en primero (Figura 1).

A pesar de que las calificaciones fueron menores en los estudiantes cuya estrategia de afrontamiento predominante fue el "pensamiento mágico", no hubo diferencias significativas al comparar la calificación con respecto a las otras estrategias. Del mismo modo, quienes utilizaron de manera predominante el "pensamiento mágico", también puntuaron más alto en la escala de fuentes de estrés, pero nuevamente las diferencias no fueron significativas con respecto al resto de las estrategias. En el cuadro 2 se muestran las calificaciones finales y los puntajes de estrés de acuerdo a la estrategia de afrontamiento predominante.

\section{Discusión}

Se observó que los niveles de estrés son constantes en los alumnos de primero y segundo años de la carrera de medicina; sin embargo, las fuentes que lo generan, así como el modo de afrontarlas, se modifican conforme transcurre el ciclo escolar y parecen asociarse al desempeño académico de esta población. El presente trabajo buscó vincular las fuentes de estrés, el afrontamiento y el desempeño académico en los estudiantes médicos, quienes se caracterizan por presentar altos niveles de

Figura 1. Porcentaje promedio de utilización de cada estrategia de afrontamiento en primero (2008) y segundo año (2009).

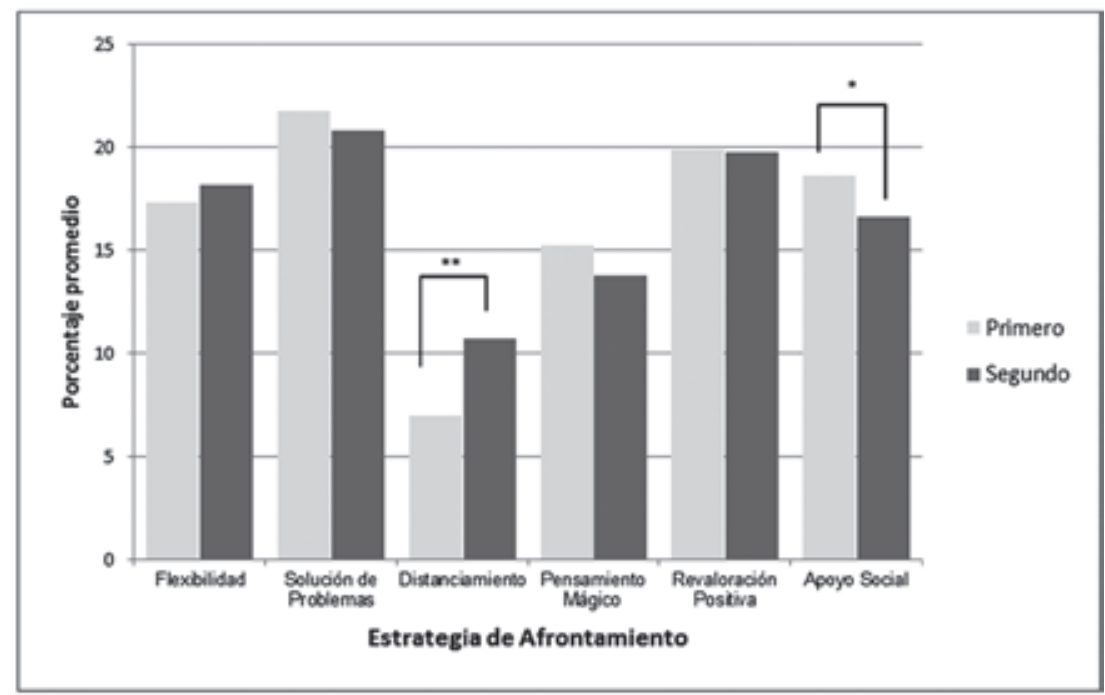

$* \mathrm{p} \leq 0.01, * * \mathrm{p} \leq 0.0001$ 
Cuadro 2. Tipos de afrontamiento de acuerdo al nivel de estrés y la calificación final de los alumnos de primero (2008) y segundo años (2009).

\begin{tabular}{|c|c|c|c|c|c|c|}
\hline & Flexibilidad & $\begin{array}{c}\text { Solución de } \\
\text { Problemas }\end{array}$ & $\begin{array}{c}\text { Pensamiento } \\
\text { mágico }\end{array}$ & $\begin{array}{c}\text { Revaloración } \\
\text { positiva }\end{array}$ & Apoyo social & \multirow[t]{2}{*}{$\mathrm{p}$} \\
\hline & \multicolumn{5}{|c|}{ Promedio ( $\pm \mathrm{DE})$} & \\
\hline Calificación Final a & $\begin{array}{c}8.37 \\
(0.11)\end{array}$ & $\begin{array}{c}8.11 \\
(1.03)\end{array}$ & $\begin{array}{c}7.46 \\
(2.53)\end{array}$ & $\begin{array}{c}8.52 \\
(0.41)\end{array}$ & $\begin{array}{c}8.47 \\
(0.53)\end{array}$ & 0.16 \\
\hline Estrés b & $\begin{array}{l}43.00 \\
(8.72)\end{array}$ & $\begin{array}{l}46.78 \\
(9.09)\end{array}$ & $\begin{array}{l}50.50 \\
(9.11)\end{array}$ & $\begin{array}{c}47.07 \\
(13.32)\end{array}$ & $\begin{array}{c}44.71 \\
(12.31)\end{array}$ & 0.85 \\
\hline
\end{tabular}

a La calificación está basada en una escala de 0 a 10.

b El puntaje de esta escala va de 24 a 120.

Nota: El distanciamiento no fue utilizado de manera predominante por ninguno de los estudiantes, por lo que se omitió de este análisis.

estrés. Los hallazgos podrían explicar por qué el estrés impacta de diferente forma, el rendimiento académico de los estudiantes médicos en los primeros dos años.

Los puntajes de las fuentes de estrés no mostraron diferencias significativas entre los dos años en los que se evaluó a los estudiantes, y pese a que este estudio no permite la comparación con el resto de los años de la carrera, hay consenso en el hecho de que los dos primeros son los más estresantes (18-20), además de que en ellos ocurren los más altos niveles de deserción (21).

Se observó que los niveles de estrés se relacionaron con el rendimiento académico del primer año, pero no del segundo, lo que generó el interés en evaluar al afrontamiento como un factor que puede modificar esta relación. A lo largo del año académico, hubo un aumento en el malestar generado por fuentes de estrés académicas, como los exámenes, las calificaciones y los hábitos de estudio; pero también cambiaron las estrategias de afrontamiento, como fue el caso de la "búsqueda de apoyo social" que presentó una disminución en su uso para el segundo año, hallazgos que son congruentes con estudios en los que el estrés y sus consecuencias se modifican de acuerdo al afrontamiento. De tal modo que, en un estudio realizado en estudiantes médicos de primer año se observó que el estrés académico se incrementaba conforme transcurría el año escolar, y también aumentaban los niveles de ansiedad y depresión cuando estaban presentes estrategias de evitación (22). En el presente trabajo, cuando los alumnos iniciaban el primer año, les preocupaba más "tener que limitar las actividades recreacionales y los compromisos sociales" y utilizaban más la estrategia de "búsqueda de apoyo social" con respecto al segundo año. Y en el inicio del segundo año, la mayor preocupación se centraba en la "proximidad de los exámenes departamentales" y utilizaban más la estrategia de "distanciamiento", es decir, el alejamiento de amigos, familiares, compañeros y personas significativas. Sin descartar que también los factores relacionados con la salud, los problemas nutricionales, el ejercicio, el sueño y el apoyo social, los que influyen sobre el rendimiento académico y crean desajuste psicológico en los estudiantes (23-26).

Por otro lado, se encontró que la estrategia de afrontamiento que utilizaron más los alumnos tanto en primero como en segundo año, fue la "solución de problemas" que consiste en generar estrategias cognitivas y conductuales encaminadas a eliminar el estrés, modificando la situación que lo produce. Por el contrario, la estrategia menos utilizada por los estudiantes tanto en primero como en segundo año fue el "distanciamiento", que se incrementó significativamente durante el segundo año. Un estudio semejante, en el que también se evaluaron los estilos de afrontamiento y sus efectos sobre la satisfacción escolar y el rendimiento académico, en estudiantes universitarios de primero y segundo año (27), encontró que la mayor parte de los estudiantes adoptaron el estilo "enfocado al problema", cuyos puntajes disminuyeron a través del tiempo pero no fueron estadísticamente significativos. También se encontraron que los estilos de afrontamiento "orientados al problema", correlacionaron positivamente con la satisfacción y con los puntajes de los exámenes prácticos, mientras que los estilos "enfocados a la emoción" mostraron una correlación negativa. No obstante, en el presente estudio, el uso de alguno de los tipos de afrontamiento no influyó sobre el desempeño académico, quizá como resultado del tamaño de la muestra y por ser alumnos de alto rendimiento (con diferencias mínimas en las calificaciones). Ésto debido a la deserción de los 
alumnos, relacionado con las bajas calificaciones que les impiden alcanzar el promedio para mantenerse en él, lo que no se controló en este estudio.

Los estudios sobre la asociación entre las fuentes de estrés y el desempeño académico de los universitarios, se ha relacionado con distintos estresores, como la carga académica de los planes de estudio, los problemas financieros, las relaciones con los compañeros, la saturación en las aulas y la ansiedad, que pueden ser considerados como indicadores potenciales que afectan el rendimiento académico (28-30). Los resultados de este estudio enfatizan la relación entre el estrés y el rendimiento académico en los alumnos de primer año, a diferencia de los de segundo, en quienes no se encontró relación con los niveles de estrés (sólo con fuentes de estrés específicas). Lo anterior puede explicarse de acuerdo al modelo psicológico del estrés propuesto por Lazarus y Folkman (1986), que señala que no todas las personas reaccionan de forma similar ante la misma fuente de estrés, ya que un estímulo puede ser percibido como positivo, negativo o neutro por el sujeto que evalúa las consecuencias de dicho estímulo y posteriormente lo revalora con base en sus capacidades de afrontamiento para poder superar dicha condición, generándose así respuestas fisiológicas, cognitivas, emocionales o conductuales que desencadenan el estrés. En este trabajo, de acuerdo a este modelo, se esperaba que el estrés influyera sobre el rendimiento académico en función del tipo de afrontamiento. Sin embargo, los resultados sólo mostraron una tendencia sin poder afirmar esta relación, aunque los autores tampoco descartan que el afrontamiento sea un mediador entre el estrés y el desempeño académico. Por lo anterior, se requiere profundizar en el estudio de esta relación, superando las limitaciones metodológicas

Las fortalezas de este estudio se derivan del diseño longitudinal que permitió dar seguimiento a los alumnos de este programa durante dos años, además de contar con participación de la mayor parte de los estudiantes del programa. Entre sus limitaciones están el sesgo de selección, dado que al ser un programa de alto desempeño, no permite generalizar los resultados a los estudiantes del programa tradicional. Además debido a qué son pocos grupos PAEA (y por tanto el tamaño de la muestra es limitado) hubo pérdidas de casi $14 \%$ de los alumnos para el segundo año. Pese a lo anterior, su diseño permitió observar la relación entre el estrés, afrontamiento y el desempeño académico.
Los diferentes estudios señalan que las fuentes de estrés en los estudiantes de medicina dependen de varios factores como el estado de salud, la ansiedad, los problemas económicos, etc. Así como de otros factores relacionados con los aspectos académicos como la carga de trabajo, el plan curricular, el tiempo que dedican a la preparación de clases y el detrimento en las actividades de esparcimiento, condiciones a las que el alumno deberá adaptarse a lo largo de la carrera. Por lo que las instituciones deben vigilar y atender estas dificultades, que enfrentan los estudiantes universitarios durante su formación académica, así como fomentar el uso de estrategias de afrontamiento adecuadas para mejorar el desempeño de los estudiantes.

\section{Agradecimientos}

Proyecto financiado por PAPIME, Proyecto No. 089-2008.

Los autores desean reconocer la participación de la Dra. Mónica Beatriz Aburto Arciniega al otorgar apoyo en los trámites administrativos y la referencia de alumnos.

Se declara que en este trabajo no hay conflicto de intereses entre los autores.

\section{Referencias}

1. Dyrbye LN, Thomas MR, Shanafelt TD. Medical student distress: causes, consequences, and proposed solutions. Mayo Clinic proceedings. Mayo Clinic. Dic 2005; 80(12): 1613-1622.

2. Tyssen R, Vaglum P, Gronvold NT, Ekeberg O. Factors in medical school that predict postgraduate mental health problems in need of treatment. A nationwide and longitudinal study. Medical education. Feb 2001; 35(2): 110-120.

3. Mosley TH, Jr., Perrin SG, Neral SM, Dubbert PM, Grothues CA, Pinto BM. Stress, coping, and well-being among third-year medical students. Academic medicine : journal of the Association of American Medical Colleges. Sep. 1994; 69(9): 765-767.

4. MacLeod RD, Parkin C, Pullon S, Robertson G. Early clinical exposure to people who are dying: learning to care at the end of life. Medical education. Ene. 2003; 37(1): 51-58.

5. Fouilloux C, Petra I, Romero M, González L. Fuentes de estrés en estudiantes de medicina. Un estudio piloto. Rev Fac Med UNAM. 1994; 37(3): 132-136.

6. Dahlin M, Joneborg N, Runeson B. Stress and depression among medical students: a cross-sectional study. Medical education. Jun. 2005; 39(6): 594-604.

7. Smith CK, Peterson DF, Degenhardt BF, Johnson JC. Depression, anxiety, and perceived hassles among entering medical students. Psychology, health \& medicine. Ene. 2007; 12(1): 31-39.

8. Üner S, Öscebe H, Telatar G, Tescan S. Assessment of Mental Health of University Students with GHQ-12. Turk J Med Sci. 2008; 38(5): 437-446.

9. Hernández-Pozo M, Coronado A, Araujo C, Cerezo R. Desempeño académico de universitarios en relación escolar con ansiedad escolar y auto-evaluación. Act. Colom. Psicol. 2008; 11(1): $13-23$ 
10. Redwood SK, Pollak MH. Student-led stress management program for first-year medical students. Teaching and learning in medicine. Winter 2007; 19(1): 42-46.

11. Yusoff MSB, Yen Yee L, Heng Wei L, et al. A study on stress, stressors and coping strategies among Malaysian medical students. Vol 12011.

12. Rosenthal TL, Rosenthal RH, Edwards NB. Students' self-ratings of stress in medical school: a replication across 20 months. Behaviour research and therapy. 1990; 28(2): 171-173.

13. Krypel MN, Henderson-King D. Stress, coping styles, and optimism: are they related to meaning of education in students' lives? Social Psychology of Education. 2010; 13(3): 409-424.

14. Hamilton S, Fagot BI. Chronic stress and coping styles: a comparison of male and female undergraduates. Journal of personality and social psychology. Nov. 1988; 55(5): 819-823.

15. Brougham R, Zail C, Mendoza C, Miller J. Stress, Sex Differences, and Coping Strategies Among College Students. Current Psychology. 2009; 28(2): 85-97.

16. Pritchard ME, Wilson GS. Do coping styles change during the first semester of college? The Journal of social psychology. Feb. 2006; 146(1): 125-127.

17. Sotelo CM, Maupone V. Traducción y estandarización del cuestionario modos de afrontamiento al estrés de Lazarus y Folkman, para una población de adolescentes mexicanos. México, D.F.: Facultad de Psicología, Universidad Nacional Autónoma de México; 2000.

18. Dyrbye LN, Thomas MR, Shanafelt TD. Systematic review of depression, anxiety, and other indicators of psychological distress among U.S. and Canadian medical students. Academic medicine: journal of the Association of American Medical Colleges. Abr. 2006; 81(4): 354-373.

19. Shah M, Hasan S, Malik S, Sreeramareddy CT. Perceived stress, sources and severity of stress among medical undergraduates in a Pakistani medical school. BMC medical education. 2010; $10: 2$.

20. Rosal MC, Ockene IS, Ockene JK, Barrett SV, Ma Y, Hebert JR. A longitudinal study of students' depression at one medical school.
Academic medicine : journal of the Association of American Medical Colleges. Jun. 1997; 72(6): 542-546.

21. Lopez-Barcena J, Gonzalez-de Cossio Ortiz M, Avila-Martinez I, Teos-Aguilar O. Epidemiological health factors and their relationship with academic performance during the first year of medical school. Study of two generations. Gaceta medica de Mexico. Mar.-Abr. 2009; 145(2): 81-90.

22. Stewart MJ, Hirth AM, Klassen G, Makrides L, Wolf H. Stress, coping, and social support as psychosocial factors in readmissions for ischaemic heart disease. International journal of nursing studies. Abr. 1997; 34(2): 151-163.

23. Trockel MT, Barnes MD, Egget DL. Health-related variables and academic performance among first-year college students: implications for sleep and other behaviors. Journal of American college health: J of ACH. Nov. 2000; 49(3): 125-131.

24. Leslie B. Hammer, Grigsby TD, Woods S. The Conflicting Demands of Work, Family, and School Among Students at an Urban University. The Journal of Psychology: Interdisciplinary and Applied 1998; 132(2): 220-226.

25. Abdulghani HM, Alrowais NA, Bin-Saad NS, Al-Subaie NM, Haji AM, Alhaqwi AI. Sleep disorder among medical students: relationship to their academic performance. Medical teacher. 2012; 34 Suppl 1: S37-41.

26. Lund HG, Reider BD, Whiting AB, Prichard JR. Sleep patterns and predictors of disturbed sleep in a large population of college students. The Journal of adolescent health : official publication of the Society for Adolescent Medicine. Feb. 2010; 46(2): 124-132.

27. Alimoglu MK, Gurpinar E, Mamakli S, Aktekin M. Ways of coping as predictors of satisfaction with curriculum and academic success in medical school. Advances in physiology education. Mar. 2011 ;35(1): 33-38.

28. Fairbrother K, Warn J. Workplace dimensions, stress and job satisfaction. Journal of Managerial Psychology. 2003; 18(1): 8-21.

29. Ongori $\mathrm{H}$. A review of the literature on employee turnover. African Journal of Business Management. 2007; 1(3): 49-54.

30. Blumberg P, Flaherty JA. The influence of noncognitive variables in student performance. Journal of medical education. Sep. 1985; 60(9): 721-723. 